\title{
Estabilidad en la población de tallos en cultivares de Urochloa con diferente intensidad de corte
}

\section{Stability in stem population in Urochloa cultivars with different cutting intensity}

Fernando Lucio-Ruiz [0]

Jonathan R. Garay-Martinez $z^{2}$ (]),

Yuridia Bautista-Martínez $z^{3}[0$

Benigno Estrada-Drouaillet 10

Filogonio J. Hernández-Guzmán 4 [0]

Andrés G. Limas-Martínez:

Santiago Joaquín-Cancino ${ }^{1 *}$

${ }^{1}$ Facultad de Ingeniería y Ciencias, Universidad Autónoma de Tamaulipas, Centro Universitario, CP. 8700. Cd. Victoria, Tamaulipas, México.

${ }^{2}$ Campo Experimental Las Huastecas, Instituto Nacional de Investigaciones Forestales, Agrícolas y Pecuarias. Carretera Tampico-Mante km 55 Villa Cuauhtémoc, CP. 89610. Altamira, Tamaulipas, México.

${ }^{3}$ Facultad de Medicina y Veterinaria, Universidad Autónoma de Tamaulipas. $\mathrm{Km} \mathrm{5}$. Carretera Cd. Victoria a Cd. Mante s/n. Cd. Victoria, Tamaulipas, México.

${ }^{4}$ Universidad Politécnica Francisco I. Madero, Domicilio Conocido $\mathrm{s} / \mathrm{n}, \mathrm{CP}$. 42660. Tepatepec, Hidalgo, México.

*Autor de correspondencia: sjoaquin@docentes.uat.edu.mx

Artículo científico

Recibido: 04 de marzo 2021

Aceptado: 01 de octubre 2021

Como citar: Lucio-Ruiz F, GarayMartínez JR, Bautista-Martínez Y, Estrada-Drouaillet B, HernándezGuzmán FJ, Limas-Martínez AG, Joaquín-Cancino S (2021) Estabilidad en la población de tallos en cultivares de Urochloa con diferente intensidad de corte. Ecosistemas y Recursos Agropecuarios Núm. Esp. II: e2960. DOI: 10.19136/era.a8nll.2960
RESUMEN. El objetivo de este estudio fue evaluar dos intensidades de corte (fija; $15 \mathrm{~cm}$ en Mulato II y Cayman y $20 \mathrm{~cm}$ en Xaraés y al $50 \%$ de la altura de planta promedio) sobre la estabilidad poblacional de tallos en cultivares del género Urochloa (Mulato II, Cayman y Xaraés). Las variables evaluadas fueron densidad, tasas de aparición, muerte y sobrevivencia e índice de estabilidad de tallos. Los resultados muestran que la densidad fija permite obtener 16, 24 y $6 \%$ mayor densidad poblacional de tallos en comparación con la intensidad al 50\% $(\mathrm{P}<0.05)$ para Mulato II, Cayman y Xaraés. En respuesta a las tasas de aparición y muerte, la intensidad fija permite mantener la población de tallos en equilibrio con índices cercanos a 1 en los tres cultivares, por lo que favorece la persistencia de la pradera a través del tiempo.

Palabras clave: Densidad poblacional, manejo, sobrevivencia, persistencia, forraje.

ABSTRACT. This study evaluated two cutting intensities (fixed; $15 \mathrm{~cm}$ in Mulato II and Cayman and $20 \mathrm{~cm}$ in Xaraés and $50 \%$ of the average plant height) on the population stability of stems in cultivars of the genus Urochloa (Mulato II, Cayman and Xaraés). The variables evaluated were density, rates of appearance, death and survival and stems stability index. The highest population density of stems was presented in the fixed intensity with 2333.66, 2500.77 and 2119.59 stems $\mathrm{m}^{-2}$, with survival rates between 89.18 and $92.45 \%$ and stability indices greater than 0.95 and less than 1.05 , that is, the intensity of fixed cut of $15 \mathrm{~cm}$ for Mulato II and Cayman and $20 \mathrm{~cm}$ for Xaraés favors the stability of the meadow increasing the persistence over time.

Key words: Population density, management, survival, persistence, forage. 


\section{INTRODUCCIÓN}

La producción de tallos es la parte esencial de la evolución de los pastos para lograr adaptación y sobrevivencia al medio en el que habitan (Rueda et al. 2018); esta característica define la persistencia de la pradera, la cual, depende de la capacidad de las plantas para sustituir los tallos muertos por nuevos y mantener la densidad poblacional (Ramírez et al. 2011). La variación en la población de tallos afecta directamente a la producción de forraje, dado que está determinada por el número de tallos y el peso individual de los mismos, comprometiendo además la persistencia de la pradera (Rojas et al. 2017). Esta dinámica está influenciada directamente por los patrones estacionales mediante los procesos de formación, muerte y supervivencia de los tallos (Ramírez et al. 2020).

La producción de nuevos tallos es un proceso continuo y acelerado por la defoliación de la planta en respuesta a la mejor recepción de luz a la base del dosel (Costa et al. 2018), mientras que, la muerte de los tallos es resultado de la eliminación de meristemos apicales necesarios realizar la formación de nuevos tallos (Ramírez et al. 2010), así como también el efecto del sombreado de las plantas de mayor altura sobre las más pequeñas, las cuales disminuyen el proceso fotosintético favoreciendo la muerte de los tallos situados en las partes inferiores (Flores et al. 2018).

El manejo agronómico realizado en las praderas, definen el comportamiento de estas, por ello, es importante considerar factores como intervalo e intensidad de cosecha (Hernández et al. 2012), ya que estos determinarán la eliminación, regeneración y ahijamiento de tallos nuevos, siempre y cuando, las condiciones ambientales así lo permitan (Rueda et al. 2018), como la temperatura, la radiación fotosintéticamente activa (calidad de la luz), humedad y nutrientes del suelo (Doust 2007). La intensidad de cosecha determina la cantidad de materia vegetal remanente en el área, misma que favorece rebrote de nuevas hojas o la formación de nuevos tallos con la ayuda de las reservas orgánicas (carbohidratos) y con este mecanismo favorecer el rebrote, la pro- ducción de forraje y asegurar la persistencia de la pradera (Rincón 2011). Por lo tanto, el objetivo de este estudio fue determinar el efecto de la intensidad de corte sobre la estabilidad poblacional de tallos en cultivares del género Urochloa.

\section{MATERIALES Y MÉTODOS}

\section{Localización del sitio experimental y característi- cas edafoclimáticas}

El estudio se realizó del 10 de junio al 21 de diciembre de 2019 en la Posta Zootécnica Ingeniero Herminio García González, ubicada en el municipio de Güémez, Tamaulipas, México (23 $56^{\prime}$ ' 17.55" LN, $99^{\circ} 06^{\prime} 2.45^{\prime \prime}$ LO), a 167 msnm. El clima del lugar es de tipo semiárido, BS1 ( $h$ ')hw con lluvias en verano y hasta $10 \%$ en invierno (Vargas et al. 2007). Se obtuvieron los datos de precipitación y temperatura de la estación meteorológica del Centro Nacional de Innovación y Transferencia de Tecnología en Agricultura de Precisión de la Facultad de Ingeniería y Ciencias perteneciente a la Universidad Autónoma de Tamaulipas, ubicada en el sitio experimental. El suelo es de textura arcillosa $(11.3,23.3$ y $65.4 \%$ de arena, limo y arcilla, respectivamente), con $\mathrm{pH}$ de 8.3 , relación de adsorción de sodio es de $0.19,4.2 \%$ de materia orgánica, $0.25 \%$ de $\mathrm{N}$ y $7.4,288.6,1.4$ y $0.5 \mathrm{mg} \mathrm{kg}^{-1}$ de $\mathrm{P}, \mathrm{K}, \mathrm{Fe}$ y $\mathrm{Zn}$, respectivamente (Garay-Martínez et al. 2018).

\section{Manejo agronómico}

Las praderas de Urochloa híbrido Mulato II, Cayman y Xaraés con dos años de establecidas, fueron sembradas en hileras espaciadas a $0.30 \mathrm{~m}$ y de forma continua dentro de la hilera, a una densidad de siembra de $5 \mathrm{~kg} \mathrm{ha}^{-1}$. Al inicio del estudio, el 10 de junio de 2019, se realizó un corte a $15 \mathrm{~cm}$ en $\mathrm{Mu}$ lato II y Cayman y a $20 \mathrm{~cm}$ de altura en Xaraés para uniformizar las praderas y formar los tratamientos con los niveles de intensidad (fija: $15 \mathrm{~cm}$ en Mulato II y Cayman y $20 \mathrm{~cm}$ en Xaraés; y al $50 \%$ de la altura de planta promedio), después de esto en las fechas de corte para la evaluación de rendimiento se cosecharon a las intensidades correspondientes a 
cada tratamiento.

\section{Dinámica de tallos}

Cada cultivar se dividió en dos intensidades y cada una de ellas en seis áreas de $100 \mathrm{~cm}^{2}(10 \times 10$ $\mathrm{cm})$ para monitorear la dinámica de tallos formando un total de 36 unidades experimentales. Después de realizar un corte de uniformidad (10 de junio) se marcaron los tallos vivos presentes en el área de 100 $\mathrm{cm}^{2}$, delimitado mediante un cuadro fijo de alambre con anillos del mismo color, conformando la población inicial y cada cuatro semanas (fechas de muestreo) los hijuelos se marcaron con un nuevo color, conformando cada fecha una generación de tallos, mientras que los tallos muertos se eliminaron del área de muestreo y se registró el dato (Martínez et al. 2020). Los datos obtenidos se utilizaron para calcular los cambios en la densidad poblacional de tallos (DPT, tallos $\mathrm{m}^{2}$ ), tasa de ahijamiento (TA) y mortalidad de tallos (TM) entre cada fecha de evaluación con las siguientes fórmulas:

$$
\begin{gathered}
T A(\%)=\frac{\text { Número de hi juelos }}{\text { DPT muestreo anterior }} \times 100 \\
T M(\%)=\frac{\text { Número de tallos muertos }}{D P T \text { muestreo anterior }} \times 100
\end{gathered}
$$

La tasa de supervivencia de tallos (TST) se obtuvo de la siguiente manera:

$$
\operatorname{TST}(\%)=100-T M
$$

El índice de estabilidad de la población de tallos (IEP) se obtuvo con la siguiente fórmula:

$$
\operatorname{IEP}(\%)=T S T(1+T A)
$$

Donde: IEP es la relación entre la población final o actual y la población anterior de tallos observada, considerando la tasa de supervivencia de tallos y tasa de ahijamiento. Su valor indica el efecto del ahijamiento y muerte de los tallos con relación a la densidad total, para un periodo determinado, valores próximos o iguales a 1 (0.95 a 1.05), indican una población de tallos en equilibrio, y una estabilidad negativa y positiva presenta valores menores a 0.95 y mayores a 1.05 , respectivamente.

\section{Rendimiento de forraje}

Para determinar el rendimiento de forraje se seleccionaron seis áreas de $1 \mathrm{~m}^{2}$, los cuales incluían el área de muestreo de tallos, en ellos se cosechó el forraje según el tratamiento. El forraje cosechado se pesó y se colocó en una estufa de aire forzado a $65{ }^{\circ} \mathrm{C}$ hasta peso constante. Después se registró el peso seco y se estimó el rendimiento ( $\mathrm{MS} \mathrm{ha}^{-1}$ ) de materia seca para cada intensidad. La altura de forraje se midió mediante una regla graduada de $1 \mathrm{~m}$ desde la superficie del suelo hasta la parte más alta de la planta sin estirar las hojas.

\section{Análisis Estadístico}

Las variables de respuesta (DPT, TA, TM, TST e IEP) se analizaron mediante el procedimiento PROC GLM de SAS, se utilizó un análisis de varianza, con un diseño completamente al azar y arreglo factorial $(3 \times 2 \times 8)$ donde los factores de estudio fueron los cultivares (Mulato II, Cayman y Xaraés), las intensidades de corte (fija y 50\%) y las fechas de muestreo (8 fechas, cada cuatro semanas), además para la comparación múltiple de medias se realizó la prueba de Tukey $(p=0.05)$.

\section{RESULTADOS}

Hubo efecto de la intensidad de corte en cada cultivar y de las fechas de muestreo $(P<0.05)$ para la densidad de tallos, presentando diferencias en el promedio de cada intensidad por cultivar (Tabla 1), en el cual, la intensidad fija ( $15 \mathrm{~cm}$ para Cayman y Mulato II y $20 \mathrm{~cm}$ para Xaraés) presentó la mayor densidad de tallos por unidad de área y fue mayor 16,33 y $6 \%$ que la intensidad del $50 \%$ en los cultivares Mulato II, Cayman y Xaraés, respectivamente. Se presentaron diferencias entre fechas de muestreo para la intensidad fija en el cultivar Mulato II (Tabla 1), presentándose la menor densidad en el muestreo realizado el 03 de agosto y el 26 de octubre con densidades menores a los 2000 tallos $\mathrm{m}^{-2}$, caso similar 
Tabla 1. Densidad de tallos (tallos $\mathrm{m}^{-2}$ ) de cultivares de Urochloa a diferente intensidad de corte con intervalo de cuatro semanas.

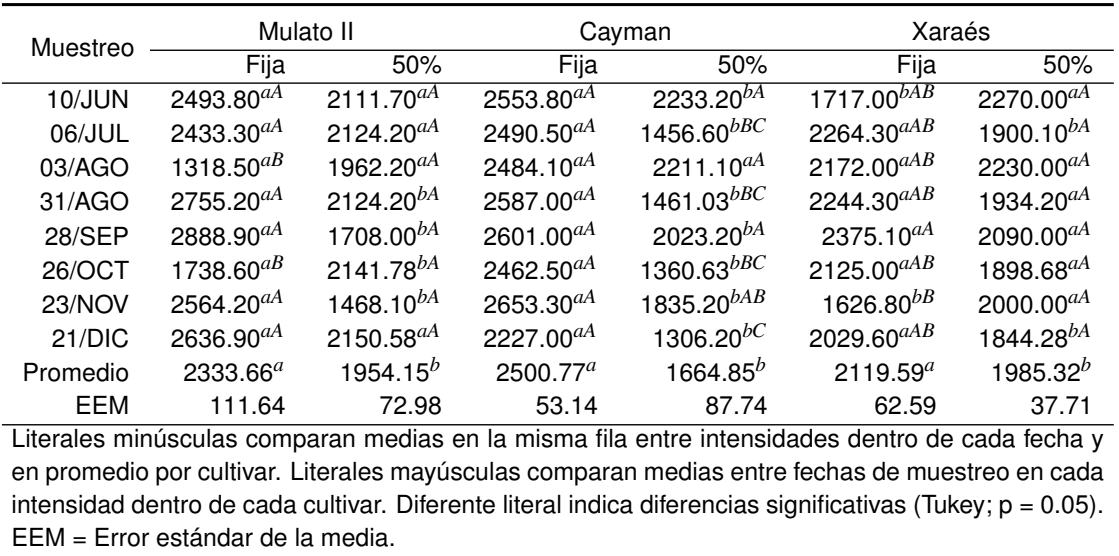

sucedió con el cultivar Xaraés con la menor densidad en el muestreo realizado el 23 de noviembre con 1626 tallos $\mathrm{m}^{-2}$, mientras que, el cultivar Cayman presentó diferencias entre fechas de muestreo en la intensidad de $50 \%$, disminuyendo de 2233 a 1306 tallos $\mathrm{m}^{-2}$ entre la primera y última fecha muestreo, por lo que las variaciones en la intensidad de corte a través del tiempo repercuten notoriamente en la densidad de tallos. Al respecto, el mantener la intensidad fija en Mulato II y Xaraés, produjo un incremento al final de la evaluación en la densidad de tallos de 5.7 y $18 \%$, respectivamente, respecto al inicio, caso contrario sucedió en Cayman el cual presentó una disminución en la densidad de $12.79 \%$ respecto a la densidad inicial (Tabla 1).

El comportamiento de la densidad de tallos es producto de las fluctuaciones en las tasas de aparición y muerte de tallos, en las que se encontró diferencias entre intensidades y fechas de muestreo por cultivar (Tabla 2, $\mathrm{P}<0.05$ ). En lo que respecta a las tasas de aparición, el cultivar Xaraés presentó diferencias en el promedio entre intensidades, en la que la intensidad fija $(20 \mathrm{~cm})$ presentó cerca de $2 \%$ más que la intensidad de $50 \%$, contrario a lo sucedido en las tasas de muerte (Tabla 2), donde la intensidad de $50 \%$ presentó la mayor tasa de muerte con $9.85 \%$. Sin embargo, entre fechas de muestreo se mantuvo actividad fluctuante en las tasas de aparición y muerte en ambas intensidades por cada cultivar $(P<0.05)$. En Mulato II, después del corte realizado el 03 de agosto cuando las alturas de las plantas fueron mayores con 36.7 y $31.9 \mathrm{~cm}$ (Figura 1) para ambas intensidades se presentaron las mayores tasas de muerte con $22.27 \%$ (Tabla 2).

Se encontraron diferencias en los promedios de las tasas de sobrevivencia para las intensidades en cada cultivar, en el cual, Mulato II y Xaraés presentaron la mayor tasa en la intensidad fija, con 89.18 y $92.47 \%$, respectivamente, mientras que, en Cayman la mayor tasa de sobrevivencia se presentó en la intensidad de $50 \%$ (Tabla 3 ). Estos resultados se ven reflejados en el índice de estabilidad de la población, en el que determina el comportamiento de la pradera respecto a la fecha anterior de muestreo considerando además la tasa de ahijamiento; durante las fechas de muestreo donde se presentaron precipitaciones menores a los $5 \mathrm{~mm}$ (03 de agosto al 26 de octubre) el índice de estabilidad se mantuvo en valores iguales o menores a 1.05 en las dos intensidades de los tres cultivares, es decir, las praderas se mantuvieron en equilibrio o presentaron riesgos de estabilidad negativa, comprometiendo la persistencia de las mismas (Tabla 3).

La intensidad de corte de $50 \%$ durante las fechas de cosecha en el cultivar Cayman fueron aproximadamente de $15 \mathrm{~cm}$, mismos que representan la intensidad de corte fija, al igual que Mulato II, mientras que Xaraés al ser el cultivar de mayor altura (Figura 1), generó intensidades de corte diferentes a la intensidad fija con $28,24,26$ y $28 \mathrm{~cm}$ para cada 
Tabla 2. Tasas de aparición y mortalidad de tallos de cultivares de Urochloa a diferente intensidad de corte con intervalo de cuatro semanas.

\begin{tabular}{|c|c|c|c|c|c|c|}
\hline \multirow{2}{*}{ Muestreo } & \multicolumn{2}{|c|}{ Mulato II } & \multicolumn{2}{|c|}{ Cayman } & \multicolumn{2}{|c|}{ Xaraés } \\
\hline & Fija & $50 \%$ & Fija & $50 \%$ & Fija & $50 \%$ \\
\hline & \multicolumn{6}{|c|}{ Tasas de aparición } \\
\hline 06/JUL & $10.33^{a A B}$ & $5.96^{b B}$ & $22.24^{a A B}$ & $15.85^{a B}$ & $20.27^{a A}$ & $15.49^{b A}$ \\
\hline 03/AGO & $2.33^{b D}$ & $8.00^{a B}$ & $10.10^{a C}$ & $17.18^{a B}$ & $9.43^{a B}$ & $12.96^{a A}$ \\
\hline 31/AGO & $0.00^{b D}$ & $2.80^{a C}$ & $0.00^{b D}$ & $1.87^{a D}$ & $0.00^{a C}$ & $0.00^{a D}$ \\
\hline 28/SEP & $6.34^{b C}$ & $21.05^{a A}$ & $7.43^{a C}$ & $9.27^{a C}$ & $7.85^{a B}$ & $1.56^{a C D}$ \\
\hline $26 / O C T$ & $9.07^{a B}$ & $7.38^{a B}$ & $12.08^{a C}$ & $10.35^{a C}$ & $7.55^{a B}$ & $4.46^{b B C}$ \\
\hline $23 / \mathrm{NOV}$ & $12.81^{a A}$ & $2.71^{b C}$ & $18.19^{a B}$ & $3.95^{a D}$ & $7.86^{a B}$ & $6.34^{a B}$ \\
\hline 21/DIC & $8.32^{a B C}$ & $1.52^{b C}$ & $24.36^{b A}$ & $41.64^{a A}$ & $1.43^{a C}$ & $1.56^{a C D}$ \\
\hline Promedio & $7.03^{a}$ & $7.06^{a}$ & $13.49^{a}$ & $14.30^{a}$ & $7.77^{a}$ & $6.05^{b}$ \\
\hline \multirow[t]{2}{*}{ EEM } & 0.95 & 1.34 & 1.81 & 2.76 & 1.36 & 1.26 \\
\hline & \multicolumn{6}{|c|}{ Tasas de muerte } \\
\hline 06/JUL & $1.02^{b E}$ & $9.62^{a B C}$ & $3.90^{b B}$ & $7.91^{a C}$ & $11.95^{a A B}$ & $15.51^{a A}$ \\
\hline 03/AGO & $11.04^{a C D}$ & $13.76^{a B}$ & $16.95^{a A}$ & $33.12^{a A}$ & $9.06^{a A B}$ & $12.51^{a A B}$ \\
\hline 31/AGO & $21.78^{a A}$ & $22.77^{a A}$ & $3.47^{a B}$ & $6.97^{a D}$ & $6.29^{b B C}$ & $9.96^{a B C}$ \\
\hline 28/SEP & $7.59^{b D}$ & $14.61^{a B}$ & $17.39^{a A}$ & $10.88^{b B C}$ & $16.21^{a A}$ & $7.72^{b C D}$ \\
\hline 26/OCT & $17.55^{a A B}$ & $4.96^{a C}$ & $6.28^{b B}$ & $12.63^{a B}$ & $0.76^{a C}$ & $8.18^{a C D}$ \\
\hline $23 / \mathrm{NOV}$ & $12.50^{a B C D}$ & $9.86^{a B C}$ & $20.69^{a A}$ & $0.74^{b D}$ & $4.51^{a B C}$ & $9.95^{a B C}$ \\
\hline 21/DIC & $13.20^{a B C}$ & $6.75^{a C}$ & $3.45^{a B}$ & $0.60^{b D}$ & $6.30^{a B C}$ & $5.12^{b D}$ \\
\hline Promedio & $12.10^{a}$ & $11.76^{a}$ & $10.30^{a}$ & $10.41^{a}$ & $7.87^{b}$ & $9.85^{a}$ \\
\hline EEM & 1.42 & 1.31 & 1.52 & 2.24 & 1.17 & 0.72 \\
\hline
\end{tabular}

Literales minúsculas comparan medias en la misma fila entre intensidades dentro de cada fecha y en promedio por cultivar.. Literales mayúsculas comparan medias entre fechas de muestreo en cada intensidad dentro de cada cultivar. Diferente literal indica diferencias significativas (Tukey; $p=0.05$ ). EEM = Error estándar de la Media.

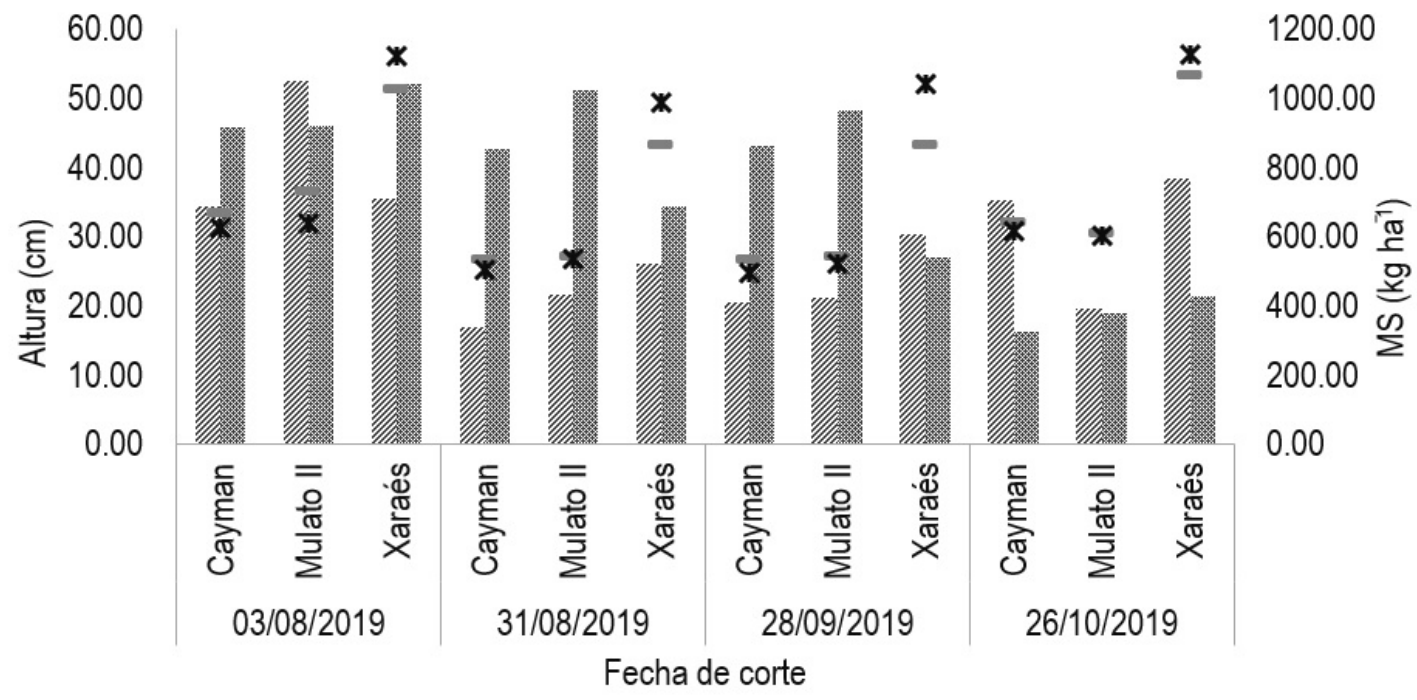

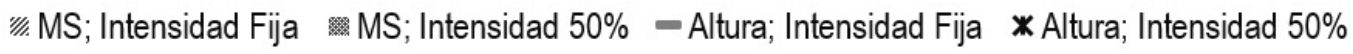

Figura 1. Producción de forraje en cultivares de Urochloa en respuesta a dos intensidades de corte en diferente fecha de corte.

una de las fechas de cosecha, resultado de la altura de planta presente antes de realizar el corte (Figura 1). Así mismo, esta variación generó $2 \%$ menos de diferencia en la tasa de aparición respecto a la inten- sidad fija $(20 \mathrm{~cm})$ (Tabla 2).

Por su parte, los rendimientos fueron más altos en la primera fecha de cosecha (Figura 1) dado a la reciente presencia de precipitaciones en 
Tabla 3. Tasas de sobrevivencia e índice de estabilidad de la población de tallos en cultivares de Urochloa a diferente intensidad de corte con intervalo de cuatro semanas.

\begin{tabular}{|c|c|c|c|c|c|c|}
\hline \multirow{2}{*}{ Muestreo } & \multicolumn{2}{|c|}{ Mulato II } & \multicolumn{2}{|c|}{ Cayman } & \multicolumn{2}{|c|}{ Xaraés } \\
\hline & Fija & $50 \%$ & Fija & $50 \%$ & Fija & $50 \%$ \\
\hline & \multicolumn{6}{|c|}{ Tasas de sobrevivencia } \\
\hline 06/JUL & $98.98^{a A}$ & $90.38^{b A B}$ & $96.10^{a A}$ & $92.09^{b B C}$ & $88.05^{a C D}$ & $84.49^{a D}$ \\
\hline 03/AGO & $88.96^{a B C}$ & $86.24^{a B C}$ & $83.05^{a B}$ & $73.03^{b D}$ & $90.94^{a B C D}$ & $87.49^{a C D}$ \\
\hline 31/AGO & $87.17^{a B C}$ & $77.23^{a D}$ & $93.03^{a A}$ & $96.53^{a A B}$ & $93.71^{a A B C}$ & $88.63^{b B C D}$ \\
\hline 28/SEP & $92.41^{a A B}$ & $83.71^{b C}$ & $82.61^{b B}$ & $90.52^{a C}$ & $83.18^{b D}$ & $92.28^{a B C D}$ \\
\hline 26/OCT & $82.41^{a C}$ & $87.87^{a A B C}$ & $93.72^{a A}$ & $87.37^{b C}$ & $99.24^{a A}$ & $92.70^{b B C D}$ \\
\hline $23 / \mathrm{NOV}$ & $87.50^{a B C}$ & $90.14^{a A B}$ & $84.23^{b B}$ & $99.26^{a A}$ & $95.49^{a A B C}$ & $90.05^{a B C D}$ \\
\hline $21 / D I C$ & $86.80^{a B C}$ & $93.25^{a A}$ & $96.55^{b A}$ & $99.40^{a A}$ & $96.69^{b A B}$ & $98.15^{a A}$ \\
\hline Promedio & $89.18^{a}$ & $86.97^{b}$ & $89.90^{b}$ & $91.17^{a}$ & $92.47^{a}$ & $90.54^{b}$ \\
\hline \multirow[t]{2}{*}{ EEM } & 1.16 & 1.12 & 1.32 & 1.77 & 1.27 & 0.87 \\
\hline & \multicolumn{6}{|c|}{ Índice de estabilidad } \\
\hline 06/JUL & $1.08^{a A}$ & $0.96^{b A}$ & $1.15^{a A}$ & $1.01^{a B}$ & $1.06^{a A B}$ & $0.98^{a A}$ \\
\hline 03/AGO & $0.97^{a B}$ & $0.93^{b A B}$ & $0.93^{a B}$ & $0.76^{b C}$ & $0.97^{a A B C}$ & $0.95^{a A B}$ \\
\hline 31/AGO & $0.78^{a C}$ & $0.84^{a B}$ & $0.93^{a B}$ & $0.95^{a B}$ & $0.94^{a B C}$ & $0.88^{a C}$ \\
\hline 28/SEP & $0.98^{a B}$ & $0.98^{a A}$ & $0.89^{b B}$ & $0.98^{a B}$ & $0.89^{a C}$ & $0.92^{a B C}$ \\
\hline 26/OCT & $0.94^{a B}$ & $0.93^{a A B}$ & $1.05^{a A}$ & $0.99^{a B}$ & $1.10^{a A}$ & $0.95^{b A B}$ \\
\hline $23 / \mathrm{NOV}$ & $0.98^{a B}$ & $0.94^{a A B}$ & $0.87^{b B}$ & $1.00^{a B}$ & $0.93^{a B C}$ & $0.96^{a A B}$ \\
\hline $21 / \mathrm{DIC}$ & $0.94^{a B}$ & $0.96^{a A}$ & $1.10^{a A}$ & $1.36^{a A}$ & $0.95^{a A B C}$ & $0.98^{a A}$ \\
\hline Promedio & $0.95^{a}$ & $0.93^{b}$ & $0.99^{a}$ & $1.01^{a}$ & $0.98^{a}$ & $0.94^{b}$ \\
\hline EEM & 0.01 & 0.01 & 0.02 & 0.03 & 0.01 & 0.00 \\
\hline
\end{tabular}

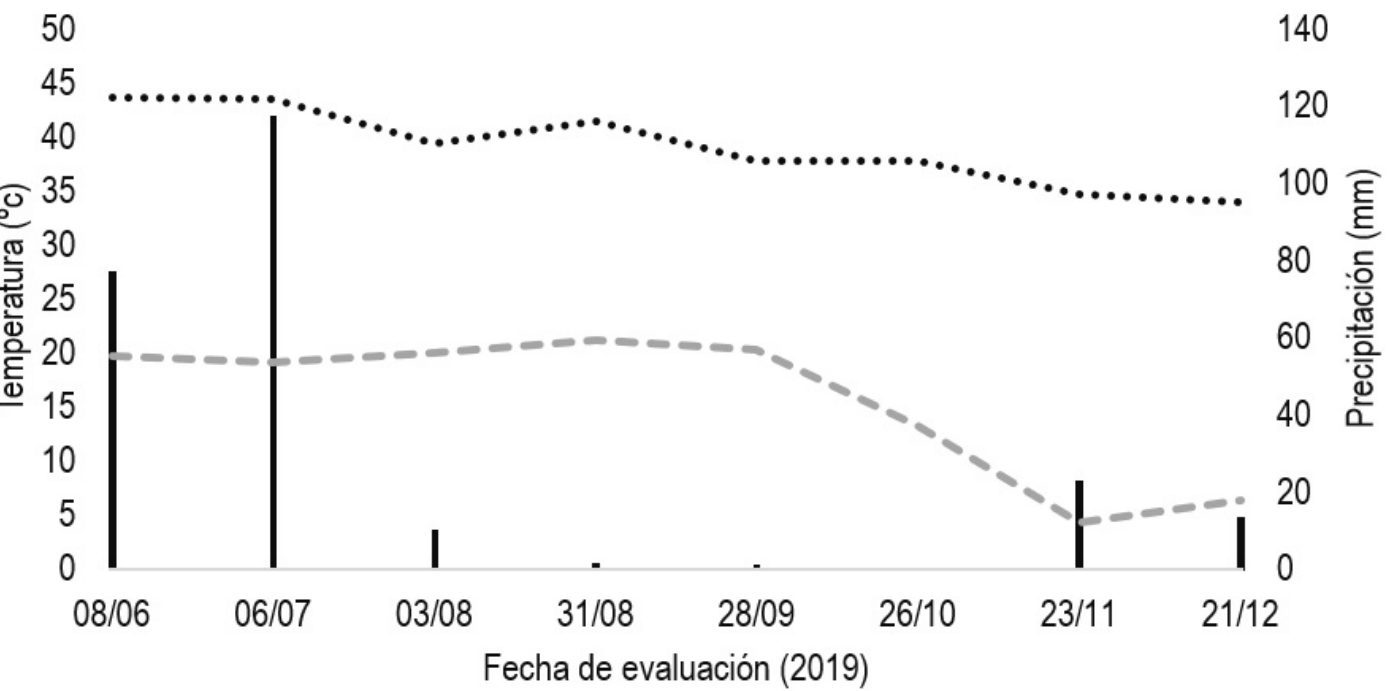

Precipitación $\quad \cdots . .$. Temp máxima $\quad$ - - - Temp mínima

Figura 2. Precipitación y temperaturas alta y baja presentes durante la fecha de evaluación.

el mes de junio y julio (Figura 2). Después de esta esa fecha, la intensidad de corte produjo cambios en el rendimiento, el cultivar Cayman disminuyó el rendimiento en la intensidad de $50 \%$ durante las siguientes fechas de muestreo, contrario a la intensidad fija, generando un incremento en el rendimiento de $52 \%$, lo mismo sucedió con Mulato II y Xaraés, en efecto, la intensidad fija genera una mejora en 
el rendimiento, resultado de la estabilidad en la población de tallos (Figura 1).

\section{DISCUSIÓN}

El comportamiento en la densidad de tallos está determinado en función del efecto de intensidad de corte y de la respuesta genética al manejo y condiciones ambientales, por lo que las intensidades constantes (15 y $20 \mathrm{~cm}$ ) a intervalos fijos (cuatro semanas) produjeron un incremento en la densidad de tallos al final de la evaluación respecto a la población inicial en Mulato II y Xaraés (Tabla 1). Al respecto, Morales et al. (2012) describen que el incremento en la densidad de tallos es una de las características que determina la diferencia entre la diversidad genética relacionada directamente con el rendimiento de forraje, es decir, a medida que la densidad aumenta, el rendimiento incrementa en una misma proporción. Pero no se deben excluir los efectos de las frecuencias e intensidades de corte o pastoreo (Hernández et al. 1999), ya que, a cortes frecuentes e intensidades severas, se reduce la competencia por luz y se produce el aumento en la densidad de tallos con hojas cortas y a intensidades ligeras se produce una mayor elongación de hojas y una disminución en la densidad de tallos (Castro et al. 2013), por lo que, la intensidad correcta permite obtener la densidad de tallos necesaria para obtener mayores rendimientos, como se presenta en Xaraés, en el cual, manejar intensidades fijas produce una respuesta en el rendimiento, incrementando más del 37 de la segunda a la última fecha de cosecha (Figura 1).

Por otro lado, la influencia de la intensidad de defoliación en la densidad de tallos, Megathyrsus maximus cv. Tanzania presenta una correlación negativa entre la intensidad de cosecha y la densidad poblacional, es decir, al disminuir la intensidad aumenta la densidad de población de tallos (Costa et al. 2018). Además, las condiciones ambientales proporcionan cambios en la densidad de población de tallos, al obtener mayores densidades durante las épocas de temperaturas optimas, por la influencia de la calidad de la luz (Flores et al. 2018).
Las condiciones ambientales apropiadas favorecen la tasa de recambio de tallos, generando incrementos en las tasas de aparición y, por ende, en la densidad poblacional de tallos (Ramírez et al. 2011), como sucedió en este estudio con Xaraés, al presentar en promedio la mayor tasa de aparición $(7.77 \%$, Tabla 2$)$ en la intensidad fija se reflejó en una mayor densidad poblacional de tallos con 2 119.59 tallos $\mathrm{m}^{-2}$. La precipitación y temperaturas registradas durante el periodo de evaluación se presentan en la Figura 2, la cual, presenta el comportamiento entre el periodo de transición de la época de máxima precipitación y la mínima. Durante el periodo de evaluación se tuvo un total acumulado de $238.59 \mathrm{~mm}$ en la precipitación, cantidad que proporciona respuesta en la formación de tallos e incremento en el rendimiento, dado que, a contenidos menores a $34 \mathrm{~mm}$ durante periodos de 60 días, especies de Brachiaria aumentan la profundidad de las raíces y disminuyen el rendimiento aéreo (Guenni et al. 2006). Las condiciones ambientales definen la respuesta de las plantas, fungiendo como factores principales la temperatura y la precipitación, en donde la temperatura toma importancia debido al efecto que tiene sobre la distribución y productividad en las plantas mediante los cambios fisiológicos producidos (Yepes y Silveira 2011). En este sentido, las gramíneas tropicales tienen como temperatura mínima de $15{ }^{\circ} \mathrm{C}$ en la cual, el cultivo detiene su crecimiento (Moreno et al. 2014).

Por otro lado, los tallos tienen un periodo de vida determinado el cual está en función principalmente de los cortes o pastoreos, como consecuencia de la eliminación de meristemos apicales a intensidades severas (Ramírez et al. 2010). Además, puede asociarse al efecto de la época en la altura de la pradera, ya que, la velocidad de crecimiento de los tallos presentes, causa mayores alturas y, por consiguiente, sombreado de las hojas y tallos situados en las partes inferiores causando su muerte (Flores et al. 2018), como se presenta en el muestreo realizado el 03 de agosto en los cultivares Mulato II y Cayman (Tabla 2), en donde presentan las mayores alturas de la pradera, mientras que, en Xaraés no se presentó esta respuesta, debido a que este cultivar 
en esta región se presenta de forma erecta, por lo tanto, no presenta niveles de sombreado que afecten a los tallos de menor altura. Al respecto, Paciullo et al. (2011) evaluaron el cultivar Xaraés en tres niveles de sombreado artificial y encontraron que a menor disposición directa del sol aumenta la tasa de elongación de los tallos, pero disminuye la cantidad de tallos por área de superficie. El mecanismo de producción de nuevos tallos es un proceso continuo y acelerado por la defoliación de la planta en respuesta a la mejor recepción de luz a la base del dosel (Costa et al. 2018). Además, la aparición de tallos en conjunto con el crecimiento individual está relacionada directamente con el rendimiento de la pradera (Castro et al. 2013).

La tasa de sobrevivencia de la población de tallos depende de la tasa de muerte que ocurre entre fechas de evaluación (Rojas et al. 2017), lo que depende entre otros factores, de las condiciones ambientales y de la densidad de plantas por área (Ramírez et al. 2020). Para este estudio, las mayores densidades fueron producidas por la implementación de las intensidades fijas, mismas que generaron los mayores porcentajes de sobrevivencia en los cultivares Mulato II y Xaraés, excepto el cultivar Cayman donde el mayor porcentaje de sobrevivencia se presentó en la intensidad de corte de $50 \%$ sobre la altura.
Los rendimientos de forraje presentes en esta evaluación se debieron principalmente por el efecto de la intensidad de cosecha, mismo efecto presentó Hernández et al. (2012), al evaluar diferente intensidad e intervalo de corte en pasto ovillo (Dactylis glomerata L.), en el cual, la intensidad ligera de corte produjo un mayor rendimiento, debido a la mayor presencia de hojas residuales, mismas que favorecen el rebrote por efecto del proceso fotosintético.

\section{CONCLUSIONES}

La estabilidad poblacional de tallos en cultivares de Urochloa híbridos (Mulato II, Cayman y Xaraés) dependen de la intensidad de cosecha aplicados. La intensidad fija de $15 \mathrm{~cm}$ para Mulato II y Cayman, y de $20 \mathrm{~cm}$ para Xaraés proporciona una mayor estabilidad poblacional de tallos, permitiendo una mayor persistencia a través del tiempo.

\section{AGRADECIMIENTOS}

Al Consejo Nacional de Ciencia y Tecnología (CONACYT) por la beca otorgada para realizar la Maestría en Ciencias Sistemas Agropecuarios y Medio Ambiente y a la Facultad de Ingeniería y Ciencias de la Universidad Autónoma de Tamaulipas.

\section{LITERATURA CITADA}

Caminha FO, Silva SC, Paiva AJ, Pereira LET, Mesquita P, Guarda VA (2010) Estabilidade da população de perfilhos de capim marandu sob lotação contínua e adubação nitrogenada. Pesquisa Agropecuaria Brasileira 45: 213-220.

Castro RR, Hernández GA, Ramírez RO, Aguilar BG, Enríquez QJF, Mendoza PSI (2013) Crecimiento en longitud foliar y dinámica de población de tallos de cinco asociaciones de gramíneas y leguminosas bajo pastoreo. Revista Mexicana de Ciencias Pecuarias 4: 201-215.

Costa LN, Jank L, Magalh JA, Magalhães JA, Rodrigues AN, Fogaça FHS, Bendahan AB, Santos FJS (2018) Características morfogênicas e estruturais de Megathyrsus maximus cv. Tanzânia sob intensidades de desfolhação. Medicina Veterinaria e Zootecnia 12: a67. DOI: 10.22256/pubvet.v12n4a67.1-7.

Doust A (2007) Architectural evolution and its implications for domestication in grasses. Annals of Botany 100: 941-950.

Flores SEJ, Hernández GA, Guerrero RJD, Vaquera HH, Cadenas S, Mendoza PSI (2018) Dinámica poblacional 
de plantas y tallos de ballico perene (Lolium perenne L.) solo y asociado con pasto ovillo (Dactulis glomerata L.) y trébol blanco (Trifolium repens L.). Acta Universitaria 28: 30-37.

Garay-Martínez JR, Joaquín-Cancino S, Estrada-Drouaillet B, Martínez-González JC, Joaquín-Torres BM, LimasMartínez AG, Hernández-Meléndez J (2018) Acumulación de forraje de pasto buffel e híbridos de Urochloa a diferente edad de rebrote. Ecosistemas y Recursos Agropecuarios 5: 573-581.

Guenni O, Gil JL, Baruch Z, Márquez L, Núñez C (2006) Respuestas al déficit hídrico en especies forrajeras de Brachiaria (Trin.) Griseb. (Poaceae). Interciencia 31: 505-5011.

Hernández GA, Martínez HPA, Zaragoza EJ, Vaquera HH, Osnaya GF, Joaquín TBM, Velazco MaEZ (2012) Caracterización de rendimiento de forraje de una pradera de alfalfa-ovillo al variar la frecuencia e intensidad de pastoreo. Revista Fitotecnia Mexicana 35: 259-266.

Hernández GA, Matthew C, Hodgson J (1999) Tiller size/density compensation in perennial ryegrass miniature swards subject to differing defoliation heights and a proposed productivity index. Grass and Forage Science 54: 347-356.

Iturbide CA (1980) Apuntes sobre pasturas vegetales. Instituto Interamericano de Ciencias Agrícolas. República Dominicana. 57p.

Martínez MD, Enríquez QJF, Esqueda EVA, Ortega JE (2020) Recambio de Tejido de hojas en Brachiaria humidicola CIAT 6133 con diferente manejo de la defoliación. Revista Mexicana de Ciencias Agrícolas 24: 47-58.

Morales NCR, Melgoza CA, Jurado GP, Martínez SM, Avendańo AC (2012) Caracterización fenotípica y molecular de poblaciones de zacate punta blanca (Digitaria californica (Benth.) Henr.). Revista Mexicana de Ciencias Pecuarias 3: 171-184.

Moreno BLS, Pedreira CGS, Boote KJ, Alves RR (2014) Base temperature determination of tropical Panicum spp. Grasses and its effects on degree-day-based models. Agricultural and Forest Meteorology 186: 26-33.

Paciullo DSC, Fernandes PB, Gomide CAM, de Castro CRT, de Souza SF, de Carvalho CAB (2011) The growth dynamics in Brachiaria species according to nitrogen dose and shade. Revista Brasileira de Zootecnia 40: 270-276.

Quero CAR, Enríquez JF, Miranda L (2007) Evaluación de especies forrajeras en América Tropical, avances o status quo. Interciencia 32: 566-571.

Ramírez RO, Hernández GA, da Silva SC, Pérez PJ, de Souza JSJ, Castro RR, Enríquez QJF (2010) Características morfogénicas y su influencia en el rendimiento de pasto mombaza, cosechado a diferentes intervalos de corte. Tropical and Subtropical Agroecosystems 12: 303-311.

Ramírez RO, Flores Al, Hernández CE, Rojas GAR, Maldonado PMA, Valenzuela LJL (2020) Dinámica poblacional de tallos e índice de estabilidad del pasto llanero. Revista Mexicana de Ciencias Agrícolas 24: 23-34.

Ramírez RO, da Silva SC, Hernández GA, Enríquez JF, Perez PJ, Quero CAR, Herrera HJG (2011) Rebrote y estabilidad de la población de tallos en el pasto Panicum maximun CV. "Mombaza" cosechado en diferentes intervalos de corte. Revista Fitotecnia Mexicana 34: 213-2020.

Rincón CA (2011) Efecto de alturas de corte sobre la producción de forraje de Brachiaria sp. en el piedemonte Ilanero de Colombia. Revista Corpoica, Ciencia y Tecnología Agropecuaria 12: 107-112.

Rojas GAR, Ventura RJ, Hernández-Garay A, Joaquín CS, Maldonado PMA, Reyes VI (2017) Dinámica poblacional de tallos de ovillo (Dactylis glomerata L.) solo y asociado con ballico perenne (Lolium perenne L.) y trébol blanco (Trifolium repens L.). Revista Mexicana de Ciencias Pecuarias 8: 419-428. 
Rueda JA, Ortega-Jiménez E, Enríquez-Quiroz JF, Palacios-Torres RE, Ramírez-Ordoñes S (2018) Tiller population dynamics in eight cultivars of elephant Grass during undisturbed growth. African Journal of Range \& Forage Science 35: 1-11.

Vargas TVM, ME Hernández R, J Gutiérrez L, CJ Plácido D y A Jiménez C (2007) Clasificación climática del Estado de Tamaulipas, México. CienciaUAT 2: 15-19.

Yepes A, Silveira BM (2011) Respuesta de las plantas ante los factores ambientales del cambio climático global (revisión). Colombia Foresta 14: 213-232. 